\title{
Decrease in prostate cancer detection during COVID-19 pandemic
}

\author{
Daniel Porav-Hodade ${ }^{1}$, Daniel Balan ${ }^{1, *}$, Raul Gherasim¹ ${ }^{1}$, Oliver Arpad Vida ${ }^{1}$, \\ Ciprian Todea-Moga ${ }^{1}$, Septimiu Voidazan², Orsolya Martha ${ }^{1}$ \\ ${ }^{1}$ Department of Urology, University of Medicine, Pharmacy, Science and Technology “George Emil Palade" of Targu Mures, 540124 \\ Targu Mures, Romania \\ 2 Department of Epidemiology, University of Medicine, Pharmacy, Science and Technology "George Emil Palade" of Targu Mures, \\ 540124 Targu Mures, Romania
}

*Correspondence: balan_dani@yahoo.com (Daniel Balan)

\begin{abstract}
Background and objectives: The aim of this study was to evaluate the impact of the COVID-19 pandemic on the detection of prostate cancer in men considered at high risk. This was done by comparing data from the Mures County Clinical Hospital, Clinic of Urology one year before (nonpandemic, NP) and during (pandemic, P) the global COVID-19 pandemic.

Methods: A retrospective study was performed based on the Mures County Clinical Hospital database. The inclusion criteria were clinical suspicion (digital rectal examination) and elevated prostate-specific antigen (PSA) level (threshold $3.2 \mathrm{ng} / \mathrm{mL}$ ). Statistical analysis was performed with STATA 11 Program. Mann-Whitney, Student and chi-square tests were used to compare median PSA, prostate volume, number of TRUSbx performed, number of core biopsies, Gleason Score values, and perineural invasion between the NP and $P$ groups.

Results: A total of 440 patients were studied, comprising 271 in the NP group and 169 in the P group. The average number of TRUSbx procedures was 22.58 per month in 2019 (NP group) and 14.08 per month in 2020 (P group), representing a decrease of $37.65 \%$. Analysis of data for the P and NP groups showed a median PSA of 10.48 vs 10.58 , mean prostatic volume of 43.69 vs 43.26 , number of core biopsies 11.39 vs 11.70, and Gleason score of 7.31 vs 7.15, respectively. Mann-Whitney $U$ test revealed no statistically significant differences between the two groups, with only the Gleason Score showing a trend for significance $(p=0.065)$.

Conclusions: The COVID-19 pandemic led to a major decrease in the number of surgeries performed, with TRUSbx declining by almost 40\%. No differences between $P$ and NP periods were observed for median PSA, prostate volume, number of core biopsies, and perineural invasion. The Gleason Score showed a slightly higher incidence of advanced prostate cancer in the P group, but this did not reach statistical significance.
\end{abstract}

\section{Keywords}

Global pandemic; COVID-19; TRUSbx; Prostate biopsy; Prostate cancer 


\section{Comparison between 2019 and 2020- TRUSbx}

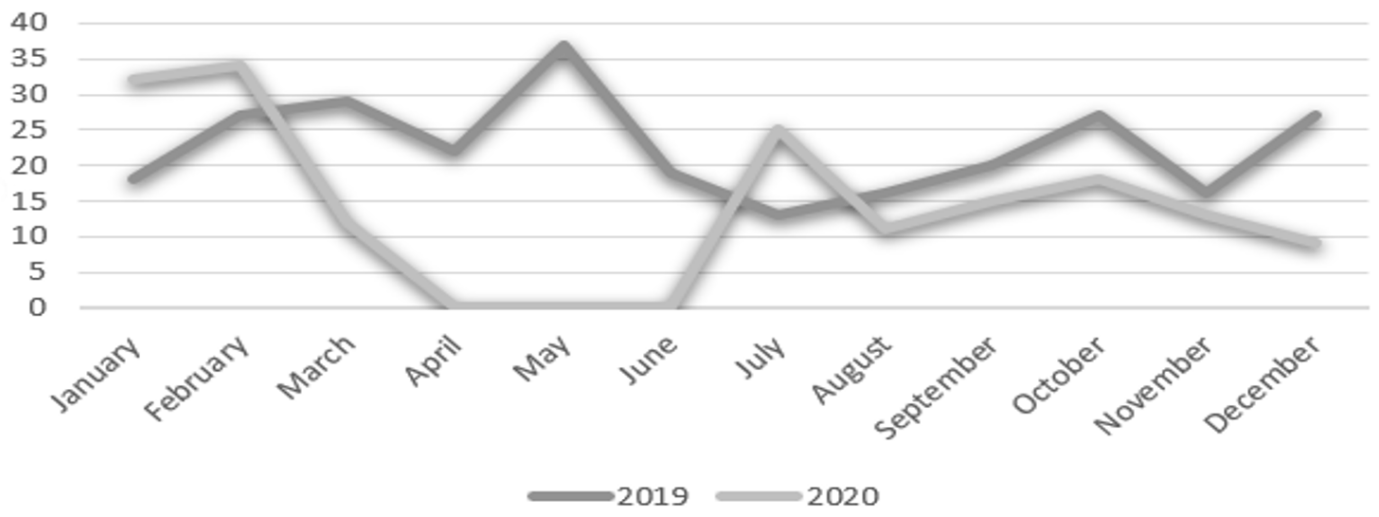

F IG. 1. Monthly comparison of TRUSbx performed in 2019 and 2020.

\section{Introduction}

Prostate cancer (PCa) is a major worldwide health concern, being the sixth most common cause of cancer-related deaths and the second most common tumor type in the male population. At the moment, transrectal ultrasound-guided prostate biopsy (TRUSbx) is the most widely used method for prostate cancer diagnosis [1-3].

A recent study by the World Health Organization (WHO), indicates that of the 19.3 million new cases of cancers reported in 2020, 1.4 million were prostate cancer, representing $7 \%$ of the total number of cases. In the central and eastern European population, the age of prostate cancer diagnosis is usually within the range of 40 to 72 years. The mortality rate from $\mathrm{PCa}$ in this region is quite high at around 13.7 deaths per 100,000 inhabitants [4, 5].

Since the first report of SARS-CoV-2 (COVID-19) infection in China on 31st of December 2019, the number of new cases worldwide increased rapidly. This has put all health care practitioners at risk and jeopardized any kind of medical act or procedure due to the risk of infection. Given the fact that older men represent the high-risk group for PCa, COVID19 infection may overlap with this population group, thus increasing the mortality. Even ignoring this overlap, a cancer diagnosis with other comorbidities may increase the risk of death from COVID-19 infection [6-10].

In accordance with EU Guidelines, the surgical activities in our Urological Department suffered from the COVID-19 pandemic, including delays in the diagnosis and staging of PCa $[6,11]$. The objective of this study was therefore to compare PCa diagnosis before and during the crisis caused by the new coronavirus in the Mures County Clinical Hospital.

\section{Materials and methods}

This retrospective study includes 440 patients who underwent TRUSbx at the Mures County Clinical Hospital, Romania, from January 2019 to December 2020. The inclusion criteria for the study were clinical suspicion upon digital rectal examination (DRE) and/or elevated prostate-specific antigen (PSA) at a threshold level of $3.2 \mathrm{ng} / \mathrm{mL}$. No age criteria were applied.

The total number of beds in the Urology Clinic before the pandemic period was 82 , serving approximately 200,000 persons. This decreased to 10 beds during the quarantine period (April-May) and then stabilized at 37 after this period. The number of confirmed cases of COVID-19 in Mures County, Romania in 2020 was 14,948 . During the quarantine period, only urological emergencies were admitted to the clinic.

The Vienna Nomogram was used for the pathological examination and most samples contained 12 randomized biopsies.

Patients were divided into two groups: nonpandemic (NP) and pandemic (P). The NP group comprised 271 patients who underwent TRUSbx in 2019, while the P group consisted of 169 patients with TRUSbx performed in 2020.

In all patients the age, PSA level, prostate volume (transrectal ultrasound assessment), number of biopsy core performed, and pathological evaluation (Gleason score and perineural invasion) were recorded.

Statistical analysis was performed with STATA 11 Statistical Program (StataCorp LLC, College Station, TX, USA). Results from biopsies performed using the same procedure before (NP) and during the global pandemic (P) were compared. Mann-Whitney, Student and chi-square tests were used to compare the Gleason score values, number of TRUSbx performed, PSA values, and the prostate volume between the NP and $\mathrm{P}$ groups.

\section{Results}

A total of 440 patients met the inclusion criteria for the study, of which 271 (62\%) were in the 2019 NP group and 169 (38\%) in the $2020 \mathrm{P}$ group. The average number of procedures for 2019 was 22.58 per month and for 2020 it was 14.08 , thus representing a decrease of $37.65 \%$ (Fig. 1).

The number of positive prostate biopsies (PCa) was 187 
T A B L E 1. Characteristics for the Pandemic and Nonpandemic groups.

\begin{tabular}{lcccccc}
\hline & & Age & PSA $(\mathrm{ng} / \mathrm{mL})$ & Prostate $\left(\mathrm{cm}^{3}\right)$ & Number of core biopsies & Gleason score \\
\hline Pandemic & Mean & 70.0296 & 79.4064 & 43.69 & 11.39 & 7.31 \\
& Std. Deviation & 7.40771 & 312.84429 & 13.63 & 3.058 & 0.885 \\
& Median & 70.0000 & 10.4800 & 40.00 & 12.00 & 7.00 \\
& Minimum & 50.00 & 0.63 & 20.00 & 3 & 6 \\
& Maximum & 87.00 & 3500.00 & 100.00 & 18 & 9 \\
Nonpandemic & Patients & 169 & 169 & 169 & 169 & 123 \\
& Mean & 76.3976 & 41.6954 & 43.26 & 2.486 & 7.15 \\
& Std. Deviation & 11.7759 & 106.38441 & 15.93 & 12.00 & 0.909 \\
Total & Median & 72.1030 & 10.5800 & 40.00 & 2 & 7.00 \\
& Minimum & 51.00 & 0.58 & 3.00 & 19 & 6 \\
& Maximum & 86.00 & 1026.00 & 100.00 & 271 & 9 \\
& Number & 271 & 271 & 440 & 440 & 310 \\
\hline
\end{tabular}

(69.0\%) for the NP group and 123 (72.78\%) in the P group, highlighting the importance of good patient selection.

The average age for the P group was 70.0 years (IQR 5087 ) and for the NP group it was 76.4 (IQR 51-86) (Table 1).

Using the same statistic test, we compared the PSA values for the same two groups. In the PANDEMIC group, the PSA maximum value was $3.500 \mathrm{ng} / \mathrm{dL}$ (mean-79.40) with $1.026 \mathrm{ng} / \mathrm{dL}$ (mean-41.69) in the NONPANDEMIC group. No major differences were recorded between the two groups regarding prostate volume (Table 1 ).

The number of confirmed cases with PCa was 123 patients in the $\mathrm{P}$ group and 187 patients in the NP group.

The Mann Whitney test was also applied to compare the Gleason score values, number of TRUSbx performed, PSA values, and the prostate volume between the P and NP groups. A p-value of 0.065 was obtained for the Gleason score (Table 2).

T A B L E 2. Mann Whitney test comparison of the Pandemic and Nonpandemic groups.

\begin{tabular}{lcccc}
\hline & PSA $(\mathrm{ng} / \mathrm{mL})$ & Prostate $\left(\mathrm{cm}^{3}\right)$ & Biopsy & Gleason score \\
\hline Mann-Whitney U & 21505.000 & 21685.000 & 21831.500 & 10035.500 \\
Wilcoxon W & 35200.000 & 58270.000 & 36027.500 & 27613.500 \\
$Z$ & -0.155 & -0.882 & -0.787 & -1.848 \\
$p$ value & 0.877 & 0.378 & 0.431 & 0.065 \\
\hline
\end{tabular}

Chi-squared tests were performed to compare pathology results between the two groups. Pathological evaluation of post-biopsy specimens revealed a higher percentage of PCa with bilateral localization (40.2\%) (Table 3).

T A B L E 3. Chi-squared test results for comparison of pathology results.

\begin{tabular}{lcccc}
\hline Diagnosis & & Pandemic & Nonpandemic & Total \\
\hline Bilateral prostate cancer & Count & 68 & 109 & 177 \\
& Percentage & $40.2 \%$ & $40.2 \%$ & $40.2 \%$ \\
\multirow{2}{*}{ Right lobe prostate cancer } & Count & 28 & 36 & 64 \\
& Percentage & $16.6 \%$ & $13.3 \%$ & $14.5 \%$ \\
Left lobe prostate cancer & Count & 27 & 42 & 69 \\
& Percentage & $16.0 \%$ & $15.5 \%$ & $15.7 \%$ \\
\hline
\end{tabular}

The chi-squared test was also used to compare the Gleason score between the $\mathrm{P}$ and NP groups (Table 4). A higher percentage of advanced-stage $\mathrm{PCa}($ Gleason $=8 /$ Gleason $=9$ ) was observed in the $\mathrm{P}$ group, but this did not reach statistical significance $(p=0.12)$. The same test revealed a higher percentage of Gleason $=6 \mathrm{PCa}$ diagnosed in the NP group.

T A B L E 4. Chi-squared for Gleason score.

\begin{tabular}{lccccc}
\hline & & & Pandemic & Nonpandemic & Total \\
\hline Gleason & 6 & Count & 17 & 40 & 57 \\
& & Percentage & $13.82 \%$ & $21.40 \%$ & $18.38 \%$ \\
& 7 & Count & 67 & 104 & 171 \\
& & Percentage & $54.47 \%$ & $55.61 \%$ & $55.16 \%$ \\
& \multirow{2}{*}{8} & Count & 22 & 18 & 40 \\
& & Percentage & $17.88 \%$ & $9.62 \%$ & $12.90 \%$ \\
& 9 & Count & 17 & 25 & 42 \\
& & Percentage & $13.83 \%$ & $13.37 \%$ & $13.56 \%$ \\
Total & & Count & 123 & 187 & 310 \\
\hline
\end{tabular}

Perineural invasion was also assessed using the chi-square test. No significant difference in the frequency of this characteristic was observed between the two groups $(p=0.20)$ (Table 5).

T A B L E 5. Chi-squared test for comparison of perineural invasion.

\begin{tabular}{|c|c|c|c|c|c|}
\hline & & & Pandemic & Nonpandemic & Total \\
\hline \multirow[t]{4}{*}{ Perineural invasion } & Yes & Count & 64 & 92 & 156 \\
\hline & & Percentage & $52.04 \%$ & $49.19 \%$ & $50.32 \%$ \\
\hline & No & Count & 59 & 95 & 154 \\
\hline & & Percentage & $47.96 \%$ & $50.81 \%$ & $49.68 \%$ \\
\hline
\end{tabular}

\section{Discussion}

Given that PCa is the second most common neoplasia in the male population, early diagnosis and treatment remain a priority.

In this study the number of patients who underwent TRUS was observed to decrease by $38 \%$ during the COVID-19 pandemic. Although the number of investigated patients was 
smaller in the P group, statistical analysis of all the data collected from the $\mathrm{P}$ and NP groups did not find any significant differences in terms of PSA, prostate volume, or number of biopsies. The only notable change was a higher Gleason score in the P group, but this did not reach statistical significance.

Ginsburg et al. [12] reported that no adverse clinical outcome was associated with a 12-month delay in surgery, even in high-risk prostatic cancers. Effective triage can minimize the negative impact of this disease during the pandemic [13].

A likely reason for the lower number of TRUSbx performed during the pandemic can be attributed to the fear of getting infected. Another obvious reason is the reduced number of beds available in hospital departments due to the high infectivity and transmission of SARS-CoV-2. The majority of medical practitioners kept the same workplace during the pandemic, while others were asked to move to a COVID19 ward [14]. At the Mures County Clinical Hospital, the urology department worked for three continuous months only with coronavirus-positive patients. This relocation had a negative impact on the diagnosis, staging, and treatment of prostate cancer patients and resulted in no TRUSbx being performed during two months (April, May) of 2020.

Compelling evidence gathered since the start of the pandemic indicates that PCa patients have a higher risk of infection than the rest of the male population [17]. Even without an oncological diagnosis, several studies have shown that more men are dying due to immunological status or smoking habit [15-17].

Prostate cancer management is currently facing a new approach. Doctors worldwide are attempting to implement an efficient triage concept that was previously discussed for application during times of disaster [18-20]. This method can screen and categorize patients, with urgent cases having priority while those described as "well" being monitored [21, 22].

Another important matter to consider is the protection for doctors and medical personnel. A recent study found that in more than $20 \%$ of SARS-CoV-2 patients with negative reverse transcriptase-polymerase chain reaction (rRT-PCR) results, viral RNA was still present in feces. This is important because without proper surgical technique, doctors performing TRUSbx could be exposed to a high risk of contracting and spreading the viral infection [23-25].

\section{Conclusions}

The COVID-19 pandemic has led to a large decrease in the number of surgeries performed, including TRUSbx.

Even though the number of TRUSbx decreased by almost $40 \%$ during the pandemic period, no differences in median PSA, prostate volume, number of core biopsies, and perineural invasion were found between pandemic and nonpandemic periods.

The Gleason Score showed a higher incidence of advanced prostate cancer in the $\mathrm{P}$ group, but this did not reach statistical significance.

\section{Author contributions}

$\mathrm{DPH}$, conceived and designed the analysis, contributed data or analysis tools. DB, conceived and designed the analysis, performed the analysis, wrote the paper. RG, collected the data, performed the analysis. SV, contributed data or analysis tools, performed the analysis. CTM, contributed data or analysis tools, designed the analysis. OVA, contributed data or analysis tools, designed the analysis. OM, conceived and designed the analysis, contributed data or analysis tools.

\section{Ethics approval and consent to participate}

All subjects gave their informed consent for inclusion before they participated in the study. The study was conducted in accordance with the Declaration of Helsinki, and the protocol was approved by the Ethics Committee of 7371/2021.

\section{Acknowledgment}

Thanks to all the peer reviewers for their opinions and suggestions.

\section{Funding}

This research received no external funding.

\section{Conflict of interest}

The authors declare no conflict of interest.

\section{References}

[1] De Nunzio C, Lombardo R, Presicce F, Bellangino M, Finazzi Agro E, Gambrosier MB, et al. Transrectal-ultrasound prostatic biopsy preparation: rectal enema vs. mechanical bowel preparation. Central European Journal of Urology. 2015; 68: 223-228.

[2] Center MM, Jemal A, Lortet-Tieulent J, Ward E, FerlayJ, Brawley O, et al. International Variation in Prostate Cancer Incidence and Mortality Rates. European Urology. 2012; 61: 1079-1092.

[3] Ismail MT, Gomella LG. Transrectal prostate biopsy. the Urologic Clinics of North America. 2013; 40: 457-472.

[4] World Cancer Research Fund International. Prostate cancer statistics. 2018. Available at: https://www.wcrf.org/dietandcancer/ prostate-cancer-statistics/ (Accessed: 27 May 2021).

[5] Schatten H. Brief Overview of Prostate Cancer Statistics, Grading, Diagnosis and Treatment Strategies. Advances in Experimental Medicine and Biology. 2018; 1095: 1-14.

[6] Dovey Z, Mohamed N, Gharib Y, Ratnani P, Hammouda N, Nair SS, et al. Impact of COVID-19 on Prostate Cancer Management: Guidelines for Urologists. European Urology Open Science. 2020; 20: 1-11.

[7] Fu L, Wang B, Yuan T, Chen X, Ao Y, Fitzpatrick T, et al. Clinical characteristics of coronavirus disease 2019 (COVID-19) in China: a systematic review and meta-analysis. Journal of Infection. 2020; 80: 656-665.

[8] Yu J, Ouyang W, Chua MLK, Xie C. SARS-CoV-2 Transmission in Patients with Cancer at a Tertiary Care Hospital in Wuhan, China. JAMA Oncology. 2020; 6: 1108.

[9] Heidenreich A, Bastian PJ, Bellmunt J, Bolla M, Joniau S, van der Kwast $\mathrm{T}$, et al. EAU guidelines on prostate cancer. part 1: screening, diagnosis, and local treatment with curative intent-update 2013. European Urology. 2014; 65: 124-137.

[10] Heidenreich A, Bastian PJ, Bellmunt J, Bolla M, Joniau S, van 
der Kwast $\mathrm{T}$, et al. EAU guidelines on prostate cancer. Part II: Treatment of advanced, relapsing, and castration-resistant prostate cancer. European Urology. 2014; 65: 467-479.

[11] Bahmad HF, Abou-Kheir W. Crosstalk between COVID-19 and prostate cancer. Prostate Cancer and Prostatic Diseases. 2020; 23: 561563.

[12] Ginsburg KB, Curtis GL, Timar RE, George AK, Cher ML. Delayed Radical Prostatectomy is not Associated with Adverse Oncologic Outcomes: Implications for Men Experiencing Surgical Delay Due to the COVID-19 Pandemic. Journal of Urology. 2020; 204: 720-725.

[13] De Vincentiis L, Carr RA, Mariani MP, Ferrara G. Cancer diagnostic rates during the 2020 'lockdown', due to COVID-19 pandemic, compared with the 2018-2019: an audit study from cellular pathology. Journal of Clinical Pathology. 2021; 74: 187-189.

[14] Wu Z, McGoogan JM. Characteristics of and Important Lessons from the Coronavirus Disease 2019 (COVID-19) Outbreak in China. Journal of the American Medical Association. 2020; 323: 1239.

[15] Smith J. Overcoming the 'tyranny of the urgent': integrating gender into disease outbreak preparedness and response. Gender \& Development. 2019; 27: 355-369.

[16] Morgan R, George A, Ssali S, Hawkins K, Molyneux S, Theobald S. How to do (or not to do)... gender analysis in health systems research. Health Policy and Planning. 2016; 31: 1069-1078.

[17] Chen N, Zhou M, Dong X, Qu J, Gong F, Han Y, et al. Epidemiological and clinical characteristics of 99 cases of 2019 novel coronavirus pneumonia in Wuhan, China: a descriptive study. The Lancet. 2020; 395: 507-513.

[18] Domenig P, Booher J, Goldman B, Greenlee J, Sircus S, Boura JA, et al. Management of Prostate Cancer During COVID-19 Pandemic: Perspective From Urologists and Radiation Oncologists in COVID Dense Metro Detroit. Cureus. 2020; 12: e9648.

[19] Mahajan V, Singh T, Azad C. Using Telemedicine during the COVID19 Pandemic. Indian Pediatrics. 2020; 57: 658-661.

[20] Mishra V. Factors affecting the adoption of telemedicine during COVID-19. Indian Journal of Public Health. 2020; 64: S234-S236.

[21] Lurie N, Carr BG. The Role of Telehealth in the Medical Response to Disasters. JAMA Internal Medicine. 2018; 178: 745-746.

[22] Hollander JE, Carr BG. Virtually Perfect? Telemedicine for Covid-19. New England Journal of Medicine. 2020; 382: 1679-1681.

23] Joshi AU, Randolph FT, Chang AM, Slovis BH, Rising KL, Sabonjian M, et al. Impact of Emergency Department Tele-intake on Left without being Seen and Throughput Metrics. Academic Emergency Medicine. 2020; 27: 139-147.

[24] Xiao F, Tang M, Zheng X, Liu Y, Li X, Shan H. Evidence for Gastrointestinal Infection of SARS-CoV-2. Gastroenterology. 2020; 158: 1831-1833.e3.

[25] Lotfi M, Hamblin MR, Rezaei N. COVID-19: Transmission, prevention, and potential therapeutic opportunities. Clinica Chimica Acta. 2020; 508: 254-266. 